\title{
Dysfunction of endothelial progenitor cells in hyperlipidemic rats involves the increase of NADPH oxidase-derived reactive oxygen species production
}

\begin{tabular}{|r|l|}
\hline Journal: & Canadian Journal of Physiology and Pharmacology \\
\hline Manuscript ID & cjpp-2016-0142.R1 \\
\hline Manuscript Type: & Article \\
\hline Date Submitted by the Author: & O2-Sep-2016 \\
\hline Complete List of Authors: & $\begin{array}{l}\text { Li, Ting-Bo; Central South University, Pharmacology } \\
\text { Zhang, Jie-Jie; Central South University, Pharmacology } \\
\text { Liu, Bin; Central South University, Pharmacology } \\
\text { Luo, Xiu-Ju; Central South University, Pharmacology } \\
\text { Ma, Qi-Lin; Xiangya Hospital Central South University } \\
\text { Peng, Jun; Central South University, Pharmacology }\end{array}$ \\
\hline Keyword: & $\begin{array}{l}\text { hyperlipidemia; NADPH oxidase; endothelial progenitor cells; reactive } \\
\text { oxygen species; Oxidized low-density lipoprotein }\end{array}$ \\
\hline
\end{tabular}

\section{SCHOLARONE}

Manuscripts 
1

2

3

4

5

6

7

8

9

\section{Dysfunction of endothelial progenitor cells in hyperlipidemic rats involves the increase} of NADPH oxidase-derived reactive oxygen species production

Ting-Bo Li ${ }^{1 *}$, Jie-Jie Zhang ${ }^{1 *}$, Bin Liu ${ }^{1}$, Xiu-Ju Luo ${ }^{2}$, Qi-Lin Ma ${ }^{3 \S}$, Jun Peng ${ }^{1,4 \S}$

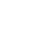

\section{Running head: NADPH oxidase and EPC dysfunction}

${ }^{1}$ Department of Pharmacology, School of Pharmaceutical Sciences, Central South University, Changsha 410078, China.

${ }^{2}$ Department of Laboratory Medicine, Xiangya School of Medicine, Central South University, Changsha 410013, China.

${ }^{3}$ Department of Cardiovascular Medicine, Xiangya Hospital, Central South University, Changsha 410008, China.

${ }^{4}$ Hunan Provincial Key Laboratory of Cardiovascular Research, School of Pharmaceutical Sciences, Central South University, Changsha 410078, China.

*Ting-Bo Li and Jie-Jie Zhang contribute equally to this work.

\section{${ }^{\S}$ Correspondence and request for reprints to:}

Professor Jun Peng, MD, PhD

Department of Pharmacology, School of Pharmaceutical Sciences, Central South University, Changsha, 410078, China.

E-mail: Junpeng@,csu.edu.cn

Or

Professor Qi-Lin Ma, MD, Department of Cardiovascular Medicine, Xiangya Hospital, Central South University, Changsha 410008, China, E-mail: mqilin2004@163.com 
1

2

3

\section{Abstract}

NADPH oxidase (NOX) is a major source of reactive oxygen species (ROS) in the body and it plays a key role in mediation of oxidative injury in cardiovascular system. The purposes of this study are to evaluate the status of NOX in EPCs of hyperlipidemic rats and to determine whether NOX- derived ROS promotes the dysfunction of endothelial progenitor cells (EPCs). The rats were fed on high-fat diet for 8 weeks to establish a hyperlipidemic rat model, which showed the increased plasma lipids and the impaired functions of circulating EPCs (including the reduced abilities in migration and adhesion), accompanied by an increase in NOX activity and ROS production. Next, EPCs were isolated from normal rats and they were treated with oxidized low-density lipoprotein (ox-LDL, $100 \mu \mathrm{g} / \mathrm{mL}$ ) for $24 \mathrm{~h}$ to induce a dysfunctional model in vitro. In agreement with our findings in vivo, ox-LDL treatment increased the dysfunctions of EPCs concomitant with an increase in NOX activity and ROS production; these phenomena were reversed by NOX inhibitor. Based on these observations, we conclude that NOX-derived ROS involves in the dysfunctions of circulating EPCs in hyperlipidemic rats and inhibition of NOX might provide a novel strategy to improve EPCs functions in hyperlipidemia.

Key words: hyperlipidemia; NADPH oxidase; endothelial progenitor cells; reactive oxygen species; oxidized low-density lipoprotein 
1

2

3

\section{Introduction}

It is well known that hyperlipidemia contributes greatly to atherosclerosis and atherosclerosis-associated diseases such as coronary heart disease, hypertension and stroke. Numerous studies from animals and humans have demonstrated that the elevated plasma lipids, such as oxidized low-density lipoprotein (ox-LDL) in particular, could induce endothelial dysfunctions of the blood vessels, initiating the first step for many cardiovascular diseases (Kim et al. 2012; Tabas et al. 2015). Under the condition of hyperlipidemia, multiple mechanisms (such as oxidative stress and inflammatory reaction) are involved in the endothelial damage in the arterial wall(Hermida and Balligand 2014; Liu et al. 2014). Following endothelial damage, the neighboring mature endothelial cells can heal the wound through proliferation and migration. However, the repairment by stem cells is needed if endothelial damage is severe (Bai et al. 2010).

Endothelial progenitor cells (EPCs) are type of stem cells primarily derived from the bone marrow. Actually, they can be isolated from umbilical cord or peripheral blood, too. EPCs have been considered as a backup system to maintain vascular endothelial integrity and to protect vascular endothelium from dysfunction(Poss et al. 2013). To date, most studies focus on the detrimental effects of hyperlipidemia or ox-LDL on endothelial cells. In fact, the impaired functions of EPCs can also occur in patients with hypercholesterolemia or in ox-LDL treated EPCs(Imanishi et al. 2003; Peng et al. 2015), suggesting that functional impairment in EPCs may contribute to endothelial abnormality in hyperlipidemia, but the underlying mechanisms remain to be elucidated.

Oxidative stress refers to a steady-state level of oxidative damage due to over 
1 production of reactive oxygen species (ROS). Growing evidence suggests that oxidative stress

2 is involved in hyperlipidemia-induced endothelial dysfunction and it can promote functional

3 impairment of EPCs (Custodis et al. 2008; Haddad et al. 2011). NADPH oxidases (NOX) -

4 derived ROS is the primary source in the cardiovascular system. There are reports that NOX

5 was activated in the aorta of hyperlipidemic mice accompanied by the dysfunction of

6 EPCs(Fenyo et al. 2011; Haddad et al. 2011). It is not known, however, whether NOX is also

7 activated in EPCs of hyperlipidemic rats and whether the NOX-derived ROS contributes to

8 the functional impairment of EPCs.

The aim of this study was to investigate the status of NOX in EPCs from hyperlipidemic rats and its association with the functions of EPCs. By using a rat model of hyperlipidemia, we measured the NOX activity of circulating EPCs and its association with EPCs functions. By using a model of ox-LDL-induced dysfunctions of EPCs as well as NOX inhibitor, we verified the role of NOX in promotion of EPCs dysfunctions.

\section{Materials and Methods}

\section{Animals}

Male Sprague-Dawley (SD) rats (120-130 g) were purchased from the Laboratory Animal Center, Xiang-Ya, School of Medicine, Central South University, China. All animals received humane care in compliance with the "Guide for the Care and Use of Laboratory Animals" published by the National Institutes of Health (NIH Publication, 8th edition, 2011), the Animal Research: Reporting In Vivo Experiments (ARRIVE) guidelines and experiments were approved by the Central South University Veterinary Medicine Animal Care and Use 
1

2

3

4

Committee.

\section{Experiments in animals}

SD rats were randomly divided into two groups ( $n=10$ per group): the control group, animals were fed on standard diet for 8 weeks; the hyperlipidemia group, rats were fed on a high-fat diet composed of $66.7 \%$ basic diet, $10 \%$ milk powder, $10 \%$ lard, $10 \%$ refined sugar, $3 \%$ cholesterol and $0.3 \%$ sodium cholate for 8 weeks. At the end of 8 weeks, rats were weighed and blood samples from all animals $(\sim 8 \mathrm{ml}$ per rat) were collected in a sterile tube containing the anticoagulant heparin for plasma or EPCs isolation under anesthesia (sodium pentobarbital, $30 \mathrm{mg} / \mathrm{kg}$, i.p.) after overnight fasting.

Measurement of plasma lipids

The levels of total cholesterol (TC), total triglycerides (TG), low-density lipoprotein (LDL) and high density lipoprotein (HDL) in plasma were assayed spectrophotometrically according to the instructions provided by a commercial diagnostic kits (Jiancheng Institute of Biotechnology, Nanjing, China). For the measurement of the level of oxidized low density lipoprotein (ox-LDL) in plasma, a commercial ELISA kit was utilized (R\&D Systems, Minnesota, USA).

\section{Characterization of endothelial progenitor cells}

EPCs were isolated by density gradient centrifugation with Ficoll-Paque ${ }^{\mathrm{TM}}$ PREMIUM (GE-Healthcare, Freiburg, Germany). After washing for three times, $10^{7}$ cells were seeded on 
1 six-well plates coated with human fibronectin (Millipore, Temecula, California, USA) in

2 endothelial basal medium-2 (Lonza, Walkersville, Maryland, USA) supplemented with

3 endothelial growth medium-2 (EGM-2) SingleQuots (Lonza, Walkersville, Maryland, USA).

4 At the $7^{\text {th }}$ day of culture, EPCs were verified by functional analysis (depending on its

5 characteristics of acetylated low-density lipoprotein incorporation and lectin binding) and

6 surface markers detection through immunofluorescence staining.

\section{Experiments in cell cultures}

9 The in vitro experiments were designed to verify the role of NOX in promotion of EPCs dysfunction. To mimic the condition of EPCs dysfunction in hyperlipidemic rats, EPCs isolated from the normal rats were incubated with ox-LDL (Yiyuan Biotech, Guangzhou, LDL is extensively oxidized by $\mathrm{Cu}_{2} \mathrm{SO}_{4}(5 \mu \mathrm{M})$ in $\mathrm{PBS}$ at $37^{\circ} \mathrm{C}$ for $20 \mathrm{~h}$, which is terminated by excess EDTA-Na2. The oxidation levels of LDL were verified by agarose gel electrophoresis for migration versus native LDL. For the ox-LDL used in this study, it migrates 2.0 fold further than the native $\mathrm{LDL}$. group, EPCs were cultured under normal conditions; the ox-LDL group, EPCs were incubated with ox-LDL (100 $\mu \mathrm{g} / \mathrm{mL})$ for $24 \mathrm{~h}$; the ox-LDL plus VAS2870 [a specific inhibitor of NOX (Cifuentes-Pagano et al. 2014), dissolved in DMSO] was added to the culture medium (50 $\mu \mathrm{M}$, final concentration) before the ox-LDL treatment; the ox-LDL plus vehicle group, equal 22 volume of vehicle (DMSO) was added to culture medium before the ox-LDL treatment. At 
1 the end of experiments, the cells were collected for functional analysis (migration and

2 adhesion) or other measurements (NOX activity and $\mathrm{H}_{2} \mathrm{O}_{2}$ content). In our preliminary

3 experiments, the effects of different concentrations of VAS2870 $(20 \mu \mathrm{M}, 50 \mu \mathrm{M}, 100 \mu \mathrm{M})$ on

4 NOX activity were evaluated and $50 \mu \mathrm{M}$ was chosen for the formal cell experiments (Data

5 not shown). Similarly, the effects of different concentrations of ox-LDL $(50 \mu \mathrm{g} / \mathrm{mL}, 100$

$6 \mu \mathrm{g} / \mathrm{mL}$ ) on EPC functions were tested and $100 \mu \mathrm{g} / \mathrm{mL}$ was chosen for the formal cell

7 experiments (Data not shown).

$9 \quad$ Functional analysis of EPCs

The functions of EPCs were evaluated by the abilities of migration and adhesion. For migration assay, EPCs were gently detached with $0.25 \%$ trypsin, $1 \times 10^{5}$ EPCs were placed in the upper chamber of a 24-well Transwell (Corning, NY, USA). $600 \mu \mathrm{L}$ EGM-2 media containing $50 \mathrm{ng} / \mathrm{mL}$ of vascular endothelial growth factor were added to the lower compartment of the chamber. After 6-h incubation at $37{ }^{\circ} \mathrm{C}$, EPCs on the top membrane was wiped off with a cotton swab. The Transwell membrane was rinsed with PBS, and then incubated in $4 \%$ paraformaldehyde at room temperature for $15 \mathrm{~min}$. For quantitation, cells were stained with $0.1 \%$ crystal violet solution (Beyotime, Nanging, China). Cells migrated into the lower chamber were counted manually in ten random fields under $\times 200$ magnification. For adhesion assay, EPCs were gently detached with $0.25 \%$ trypsin. $5 \times 10^{4}$ cells were plated in 12-well plates coated with $10 \mu \mathrm{g} / \mathrm{mL}$ human plasma fibronectin (Millipore,

21 Temecula, California, USA). After 30 min of incubation, non-adherent cells were removed by 
1

2

3

4

5

6

7

8

9

with DAPI (Beyotime, Nanjing, China). Five random fields were counted in each well under $\times 100$ magnification.

Measurement of NOX activity

The NOX activity was determined by measuring the NADPH-dependent superoxide dismutase (SOD)-inhibitable cytochrome c reduction (GENMED, Shanghai, China). Briefly, the reaction buffer $(900 \mu \mathrm{l})$ containing the NOX substrate (NADPH) and the oxidized cytochrome $\mathrm{C}$ in a quartz cuvette was incubated at $30^{\circ} \mathrm{C}$ for $3 \mathrm{~min}$, and then an aliquot $(100 \mu \mathrm{l})$ of the supernatant from EPCs lysate was added to the reaction mixture and incubated at $30{ }^{\circ} \mathrm{C}$ for $15 \mathrm{~min}$. The change of absorbance at $550 \mathrm{~nm}$ was monitored by a spectrophotometer. A buffer blank was measured in each assay, and SOD-inhibitable cytochrome c reduction in buffer blank was subtracted from each sample. The NOX activity was calculated as SOD-inhibitable cytochrome c reduction and expressed as $\mathrm{O}_{2}^{-}$in nmol/mg protein/min.

(1)

\section{Measurement of ROS production in EPCs}

To measure the generation of $\mathrm{H}_{2} \mathrm{O}_{2}$ from EPCs, $50 \mu \mathrm{L}$ of culture medium and $100 \mu \mathrm{L}$ of work solution (0.25 mM ammonium ferrous II sulfate, $25 \mathrm{mM} \mathrm{H}_{2} \mathrm{SO}_{4}, 100 \mathrm{mM}$ sorbitol, and 125 $\mathrm{mM}$ xylenol orange) were mixed and incubated at room temperature for $20 \mathrm{~min}$. The change of absorbance at $560 \mathrm{~nm}$ was monitored, and the level of $\mathrm{H}_{2} \mathrm{O}_{2}$ was calculated according to a standard curve made from the standard solutions provided by the supplier (Beyotime, Nanjing,

\section{China).}

The measurement of intracellular $\mathrm{ROS}\left(\mathrm{H}_{2} \mathrm{O}_{2}\right)$ levels was dependent on the fluorescent 
signal of 2',7'-dichlorodihydrofluorescein diacetate (DCFH-DA), a cell-permeable indicator of ROS (Beyotime, Jiangsu, China). DCFH-DA is nonfluorescent until the acetate groups are removed by intracellular ROS. Briefly, EPCs were washed with PBS and incubated with DCFH-DA $(10 \mu \mathrm{M})$ at $37^{\circ} \mathrm{C}$ for $20 \mathrm{~min}$. Then, the ROS-mediated fluorescence was observed under a fluorescent microscope with excitation set at $502 \mathrm{~nm}$ and emission set at $523 \mathrm{~nm}$. Arbitrary fluorescent units were normalized vs control and expressed as fold change.

\section{Statistical analysis}

SPSS software (version 20) was used for statistical analysis. Data were presented as means \pm SD. Differences in measured values between two groups were analyzed by Student's $t$ test while among multiple groups were analyzed by ANOVA with Bonferroni's multiple comparison tests. Differences were considered as significant when $P<0.05$.

\section{Results}

\section{Body weight and plasma lipid profile}

After 8 weeks of high-fat diet feeding, body weight of rats in the hyperlipidemia group was significantly increased compared to that in the control group (Table 1). In the hyperlipidemic rats, plasma levels of lipids including TC, TG, LDL and ox-LDL were dramatically elevated compared with that in the control rats. On the contrary, plasma level of HDL in the hyperlipidemic rats was markedly decreased compared with that in the control rats.

\section{Phenotypic characterization of endothelial progenitor cells}


1 The cultured mononuclear cells from rat peripheral blood generated EPCs as expected. At the

$27^{\text {th }}$ day of culture, the attached cells displayed previously defined functions of EPCs (lectin

3 binding, acetylated low-density lipoprotein incorporation, Figure 1, A), and expressed the

4 stem cell marker (CD34) as well as endothelial cell markers (Flk-1, CD31, CD144 and eNOS)

5 (Figure 1, B).

6

7 Dysfunction of circulating endothelial progenitor cells in hyperlipidemic rats

8 To evaluate the functions of EPCs, the abilities of migration and adhesion for EPCs were

9 measured. The results from Transwell experiments showed that the migrated EPCs numbers were dramatically reduced in the hyperlipidemia group compared with the control group (Figure 2A), accompanied by a decrease in adhesive EPCs numbers (Figure 2B).

\section{Increase of NOX activity in endothelial progenitor cells from hyperlipidemic rats}

To explore whether oxidative stress involves the dysfunction of EPCs in hyperlipidemic rats, we examined the activity of NOX, a major source of ROS in cardiovascular system. Compared with the control rats, NOX activity (Figure 3A) in EPCs from the hyperlipidemic rats was dramatically increased, accompanied by the elevated $\mathrm{H}_{2} \mathrm{O}_{2}$ levels in the culture medium (Figure 3B) as well as the intracellular ROS levels (Figure 3C).

\section{Inhibition of NOX attenuated ox-LDL-induced EPCs dysfunction in vitro}

As displayed in Figure 4, incubation of EPCs with ox-LDL $(100 \mu \mathrm{g} / \mathrm{mL})$ for $24 \mathrm{~h}$ significantly induced cellular dysfunction (a decrease in migrated numbers and adhesive number). 
1 Administration of NOX specific inhibitor (VAS2870) dramatically attenuated

2 ox-LDL-induced EPCs dysfunctions. The vehicle of VAS2870 did not have such effect.

3

4

\section{Decrease of ox-LDL-induced ROS production in EPCs by NOX inhibitor}

Consistent with the findings in EPCs from the hyperlipidemia rats, NOX activity in ox-LDL-treated EPCs was significantly increased (Figure 5A), accompanied by the elevated NOX-derived products (Figure 5, B and C); these effects were attenuated by NOX inhibitor (VAS2870). The vehicle of VAS2870 did not show such effects.

\section{Discussion}

In the present study, we explored the correlation between NOX and EPCs functions by using the hyperlipidemic rats and the ox-LDL-induced dysfunctional model of EPCs in vitro. Our results from animal and cell experiments showed that the EPCs functions (migration and adhesion) were impaired in hyperlipidemic rats or after ox-LDL treatment in vitro, accompanied by the increased NOX activity and NOX-derived products $\left(\mathrm{H}_{2} \mathrm{O}_{2}\right)$. Inhibition of NOX (by VAS2870) showed beneficial effect on ox-LDL-induced EPCs dysfunctions. To the best of our knowledge, this is the first study to provide evidence that NOX-mediated oxidative stress involves the dysfunctions of EPCs in the hyperlipidemic rats.

Abnormally high levels of blood lipids (such as cholesterol and triglycerides) and lipoproteins (such as LDL) are the characteristics of hyperlipidemia. In this study, the plasma levels of triglycerides, cholesterol, LDL and ox-LDL in rats were significantly elevated after 8 weeks of high-fat diet feeding concomitant with a decrease in HDL level (the good 
cholesterol), suggesting that we successfully established a rat model of hyperlipidemia, which is a common and major risk factor for cardiovascular diseases. It is well-recognized that the elevated plasma levels of lipids and lipoproteins can accelerate the process of atherosclerosis development via multiple pathways(Catapano et al. 2014; Ouweneel and Van Eck 2015). Among them, hyperlipidemia-induced endothelial dysfunction is thought as the first step in initiating the atherosclerosis development (Huang et al. 2013). At early stage, when the endothelial damage within the blood vessel is mild, the neighboring mature endothelial cells can heal the wound through proliferation and migration. With endothelial damage getting worse, EPCs are mobilized to take part in the repairmen of damage because they are able to differentiate into mature endothelial cells(Kawamoto and Losordo 2008; Zhang et al. 2014b). However, a number of studies from animal models or patients have demonstrated that the functions of circulating EPCs were impaired under many pathological conditions such as hypertensions and hyperlipidemia(Peng et al. 2015; Rossi et al. 2013; Zhou et al. 2010), which may compromise the capability of EPCs to heal the endothelial injury. Consistent with the reports, in this study, we have found that the functions of EPCs (migration and adhesion) were dramatically attenuated in the hyperlipidemic rats or in the ox-LDL-treated cells compared to that in the control rats or EPCs, which may provided a reasonable explanation, at least partially, for the deteriorated endothelial function in hyperlipidemic rats.

The mechanisms for the dysfunction of EPCs in hyperlipidemia are multi-facial. Accumulating evidence suggests that over-production of ROS may account for cellular dysfunction under many pathological conditions(Ellinsworth 2015; Mittal et al. 2014). A major source of ROS in the body is from NOX. The immediate product of NOX is superoxide 
1 anion, which rapidly dismutates to $\mathrm{H}_{2} \mathrm{O}_{2}$ either spontaneously or catalyzed by $\mathrm{SOD}$ (Konior et

2 al. 2014). Studies from other labs and ours have demonstrated that NOX activity was

3 up-regulated under multiple conditions, such as myocardial infarction (Zhang et al. 2015),

4 hypertension(Sasser et al. 2014), celebral ischemia and hyperlipidemia (Fenyo et al. 2011;

5 Zhang et al. 2014a). Based on these reports, we speculate that NOX-derived ROS might be

6 responsible for, at least in part, the deteriorated functions of circulating EPCs in the

7 hyperlipidemic rats. In this study, we indeed found that the activity of NOX was increased in

8 EPCs from the hyperlipidemic rats or in EPCs after the ox-LDL treatment, accompanied by an

9 elevation in NOX-derived products $\left(\mathrm{H}_{2} \mathrm{O}_{2}\right)$ as well as the deteriorated functions. The ox-LDL-induced the increase of NOX activity and EPCs dysfunctions in vitro was significantly attenuated in the presence of VAS2870, a specific inhibitor of NOX. These results suggested a cause-and-effect relationship between NOX and EPCs dysfunction.

In conclusion, the results presented in this study demonstrate for the first time that NOX-derived ROS involves in the deteriorated functions of circulating EPCs in hyperlipidemia. Thus, prevention of the NOX-derived ROS production by NOX inhibitors may provide a novel strategy to improve EPCs functions in hyperlipidemia.

\section{Limitation of this study}

There are several limitations that need to be acknowledged and addressed regarding the present study. First of all, although we established an ox-LDL-induced EPC dysfunction model in vitro, we did not provide direct evidence that ox-LDL could produce the same effects on NOX activity, ROS formation, and EPC function in vivo. Secondly, although the cell experiments 
1 support the cause- and -effect relationship between NOX and EPCs functions, it still lacks the

2 evidence in vivo. In our future studies, it is necessary to verify the in vitro results through

3 intervention of NOX in vivo. Finally, although the method for EPCs isolation is well-established

4 in our lab (Liu et al. 2016; Zhou et al. 2010), it is still necessary to further characterize the EPCs

5 by flow-cytometry.

6

\section{$7 \quad$ Acknowledgements}

8 This work was supported by the Major Research Plan of the National Natural Science

9 Foundation of China (No.91439104 to Jun Peng), National Natural Science Foundation of

China (No.81373409 to Jun Peng, No.81370250 to Qi-Lin Ma), Natural Science Foundation

of Hunan Province, China (No.13JJ2008 to Jun Peng), and Doctoral Fund of the Ministry of

Education of China (No.20120162110056 to Jun Peng).

(4)
6

\section{(1)} 9 20 21 
1

2

3

4

5

6

7

8

9

10

11

12

13

14

15

\section{References}

Bai, X. Wang, X., and Xu, Q. 2010. Endothelial damage and stem cell repair in atherosclerosis. Vascul. Pharmacol. 52 (5-6): 224-229.

Catapano, A.L. Farnier, M. Foody, J.M. Toth, P.P. Tomassini, J.E. Brudi, P., et al. 2014. Combination therapy in dyslipidemia: where are we now? Atherosclerosis, 237 (1): 319-335.

Cifuentes-Pagano, E. Meijles, D.N. and Pagano, P.J. 2014. The quest for selective nox inhibitors and therapeutics: challenges, triumphs and pitfalls. Antioxid. Redox. Signal. 20 (17): 2741-2754.

Custodis, F. Baumhakel, M. Schlimmer, N. List, F. Gensch, C. Bohm, M., et al. 2008. Heart rate reduction by ivabradine reduces oxidative stress, improves endothelial function, and prevents atherosclerosis in apolipoprotein E-deficient mice. Circulation, 117 (18): $2377-2387$.

Ellinsworth, D.C. 2015. Arsenic, reactive oxygen, and endothelial dysfunction. J. Pharmacol. Exp. Ther. 353 (3): 458-464.

Fenyo, I.M. Florea, I.C. Raicu, M., and Manea, A. 2011. Tyrphostin AG490 reduces NAPDH oxidase activity and expression in the aorta of hypercholesterolemic apolipoprotein E-deficient mice. Vascul. Pharmacol. 54 (3-6): 100-106.

Haddad, P. Dussault, S. Groleau, J. Turgeon, J. Maingrette, F., and Rivard, A. 2011. Nox2-derived reactive oxygen species contribute to hypercholesterolemia-induced inhibition of neovascularization: effects on endothelial progenitor cells and mature endothelial cells. Atherosclerosis, 217 (2): 340-349. 
1 Hermida, N., and Balligand, J.L. 2014. Low-density lipoprotein-cholesterol-induced endothelial

2

3

4 dysfunction and oxidative stress: the role of statins. Antioxid. Redox. Signal. 20 (8): 1216-1237.

Huang, Q. Qin, L. Dai, S. Zhang, H. Pasula, S. Zhou, H., et al. 2013. AIP1 suppresses atherosclerosis by limiting hyperlipidemia-induced inflammation and vascular endothelial dysfunction. Arterioscler. Thromb. Vasc. Biol. 33 (4): 795-804.

Imanishi, T. Hano, T. Matsuo, Y., and Nishio, I. 2003. Oxidized low-density lipoprotein inhibits vascular endothelial growth factor-induced endothelial progenitor cell differentiation. Clin. Exp. Pharmacol. Physiol. 30 (9): 665-670.

Kawamoto, A., and Losordo, D.W. 2008. Endothelial progenitor cells for cardiovascular regeneration. Trends Cardiovasc. Med. 18 (1): 33-37.

Kim, J.A. Montagnani, M. Chandrasekran, S., and Quon, M.J. 2012. Role of lipotoxicity in endothelial dysfunction. Heart Fail. Clin. 8 (4): 589-607.

Konior, A. Schramm, A. Czesnikiewicz-Guzik, M., and Guzik, T.J. 2014. NADPH oxidases in vascular pathology. Antioxid. Redox. Signal. 20 (17): 2794-2814.

Liu, B. Li, T. Peng, J.J. Zhang, J.J. Liu, W.Q. Luo, X.J., et al. 2016. Non-muscle myosin light chain promotes endothelial progenitor cells senescence and dysfunction in pulmonary hypertensive rats through up-regulation of NADPH oxidase. Eur. J. Pharmacol. 775: 67-77.

Liu, Y.H. Liu, Y. Chen, J.Y. Zhou, Y.L. Chen, Z.J. Yu, D.Q., et al. 2014. LDL cholesterol as a novel risk factor for contrast-induced acute kidney injury in patients undergoing percutaneous coronary intervention. Atherosclerosis, 237 (2): 453-459.

Mittal, M. Siddiqui, M.R. Tran, K. Reddy, S.P., and Malik, A.B. 2014. Reactive oxygen species in 
inflammation and tissue injury. Antioxid. Redox. Signal. 20 (7): 1126-1167.

Ouweneel, A.B., and Van Eck, M. 2016. Lipoproteins as modulators of atherothrombosis: From endothelial function to primary and secondary coagulation. Vascul. Pharmacol. 82(7):1-10

Peng, J. Liu, B. Ma, Q.L. and Luo, X.J. 2015. Dysfunctional endothelial progenitor cells in cardiovascular diseases: role of NADPH oxidase. J. Cardiovasc. Pharmacol. 65 (1): $80-87$.

Poss, J. Lorenz, D. Werner, C. Pavlikova, V. Gensch, C. Speer, T., et al. 2013. Diesel exhaust particles impair endothelial progenitor cells, compromise endothelial integrity, reduce neoangiogenesis, and increase atherogenesis in mice. Cardiovasc. Toxicol. 13 (3): 290-300.

Rossi, F. Bertone, C. Michelon, E. Bianco, M.J., and Santiemma, V. 2013. High-density lipoprotein cholesterol affects early endothelial progenitor cell number and endothelial function in obese women. Obesity (Silver Spring), 21 (11): 2356-2361.

Sasser, J.M. Cunningham, M.W., Jr., and Baylis, C. 2014. Serelaxin reduces oxidative stress and asymmetric dimethylarginine in angiotensin II-induced hypertension. Am. J. Physiol. 307 (12): F1355-1362.

Tabas, I. Garcia-Cardena, G., and Owens, G.K. 2015. Recent insights into the cellular biology of atherosclerosis. J. Cell. Biol. 209 (1): 13-22.

Zhang, H.F. Li, T.B. Liu, B. Lou, Z. Zhang, J.J. Peng, J.J., et al. 2014a. Inhibition of myosin light chain kinase reduces NADPH oxidase-mediated oxidative injury in rat brain following cerebral ischemia/reperfusion. Naunyn. Schmiedebergs. Arch. Pharmacol. 388 (9): 953-963. 
1

2

3

4

5

6

7

8

9

10

11

12

13

14

15

16

17

18

19

20

21

22

1

12 . 4

\section{6}

\section{7} 8 19

\section{0} 1 22

Zhang, M. Malik, A.B., and Rehman, J. 2014b. Endothelial progenitor cells and vascular repair. Curr. Opin. Hematol. 21 (3): 224-228.

Zhang, Y.S. Liu, B. Luo, X.J. Li, T.B. Zhang, J.J. Peng, J.J., et al. 2015. Nuclear cardiac myosin light chain 2 modulates NADPH oxidase 2 expression in myocardium: a novel function beyond muscle contraction. Basic. Res. Cardiol. 110 (4): 38.

Zhou, Z. Peng, J. Wang, C.J. Li, D. Li, T.T. Hu, C.P., et al. 2010. Accelerated senescence of endothelial progenitor cells in hypertension is related to the reduction of calcitonin gene-related peptide. J. Hypertens. 28 (5): 931-939.

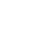

15 
Figure legends

Figure 1. Characterization of rat peripheral blood-derived endothelial progenitor cells (EPCs). A.Three-color fluorescent imaging indicating Dil-acetylated low-density lipoprotein (Dil-acLDL) incorporation (red) and FITC lectin (FITC-UEA-1) binding (green) by endothelial progenitor cells. Nuclei appear dark blue (DAPI). B. Immunofluorescence staining revealed the expression of CD34 (the stem cell marker), Flk-1, CD31, CD144 and eNOS (the endothelial cell markers) in EPCs. Nuclei were counterstained with DPI (blue).

Figure 2. Impaired functions of the circulating endothelial progenitor cells in hyperlipidemic rats. A. Left panel, representative images for EPCs migration (transwell) assay; right panel, the migrated EPC numbers. B. Left panel, representative images for EPCs adhesion assay; right panel, the adhesive EPC numbers. All values are expressed as mean \pm $\mathrm{SD}\left(n=10\right.$ per group). ${ }^{* *} P<0.01$ vs Control.

Figure 3. Elevation of NOX activity and ROS production in endothelial progenitor cells of hyperlipidemic rats. A. NOX activity in EPCs. $\mathrm{B} . \mathrm{H}_{2} \mathrm{O}_{2}$ levels in culture medium. C. Left panel, Representative image of fluorescent signal of DCFH-DA for total ROS in EPCs; right panel, statistic value for fluorescent density. All values are expressed as mean $\pm \operatorname{SD}(n=$ 10 per group). NOX, NADPH oxidase; ROS, reactive oxidative species. ${ }^{* *} P<0.01$ vs Control.

Figure 4. Impaired functions in ox-LDL-treated endothelial progenitor cells. A. Representative images for migration (transwell) and adhesion assays. B. The migrated EPC numbers C. The adhesive EPC numbers. All values are expressed as mean $\pm \mathrm{SD}(n=6$ per group). VAS2870: the NOX inhibitor; EPC, endothelial progenitor cell. ${ }^{* *} P<0.01$ vs Control; 
$1 \quad{ }^{\# \#} P<0.01$ vs ox-LDL.

2 Figure 5. Elevation of NOX activity and ROS production in ox-LDL-treated endothelial

3 progenitor cells. A. NOX activity in EPCs. B. $\mathrm{H}_{2} \mathrm{O}_{2}$ levels in EPCs. C. Left panel,

4 Representative image of fluorescent signal of DCFH-DA for total ROS in EPCs; right panel,

5 statistic value for fluorescent density. All values are expressed as mean $\pm \operatorname{SD}$ ( $n=6$ per group).

6 NOX, NADPH oxidase; ROS, reactive oxidative species. ${ }^{* *} P<0.01$ vs Control; ${ }^{\# \#} P<0.01$ vs

7 ox-LDL. 
Table 1 Body weight and plasma lipids

\begin{tabular}{lcc}
\hline & $\begin{array}{c}\text { Control } \\
(n=10)\end{array}$ & $\begin{array}{c}\text { Hyperlipidemia } \\
(n=10)\end{array}$ \\
\hline Body weight $(\mathrm{g})$ & $427.7 \pm 10.6$ & $529.6 \pm 10.2^{* *}$ \\
TC $(\mathrm{mmol} / \mathrm{L})$ & $2.20 \pm 0.21$ & $3.92 \pm 0.24^{* *}$ \\
TG $(\mathrm{mmol} / \mathrm{L})$ & $0.75 \pm 0.10$ & $1.25 \pm 0.09^{* *}$ \\
LDL $(\mathrm{mmol} / \mathrm{L})$ & $0.65 \pm 0.09$ & $2.48 \pm 0.30^{* *}$ \\
Ox-LDL $(\mu \mathrm{g} / \mathrm{L})$ & $50.9 \pm 1.21$ & $62.18 \pm 1.68^{* *}$ \\
HDL $(\mathrm{mmol} / \mathrm{L})$ & $1.22 \pm 0.11$ & $0.65 \pm 0.06^{* *}$ \\
\hline
\end{tabular}

TC: total cholesterol; TG: total triglycerides; LDL: low-density lipoprotein HDL: high density lipoprotein; ox-LDL: oxidized low density lipoprotein ${ }^{* *} P<0.01$ vs control 
$\mathbf{A}$

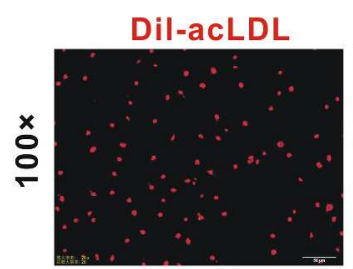

FITC-UEA-1
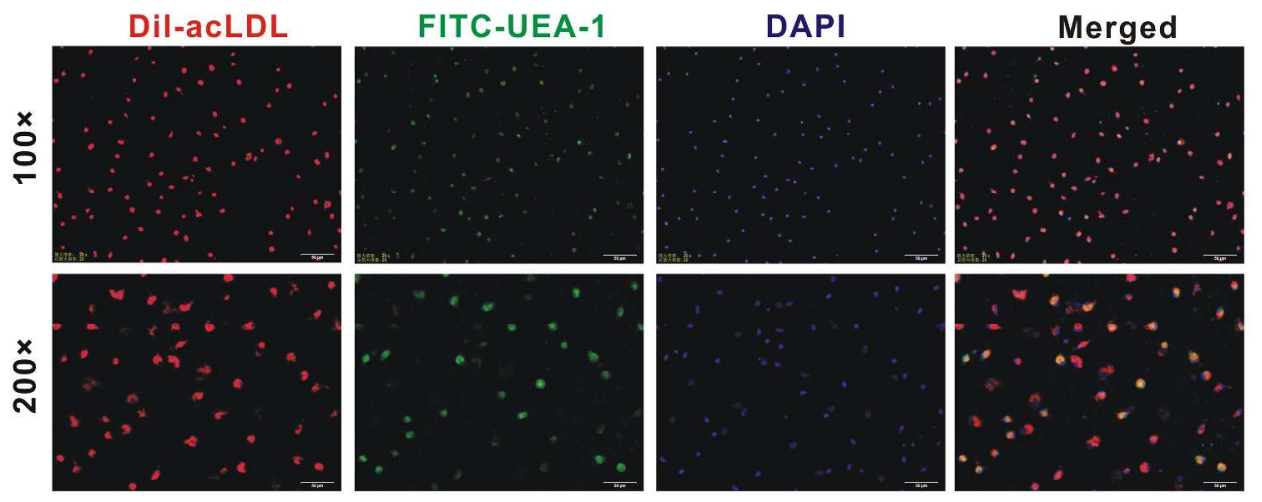

B

eNOS

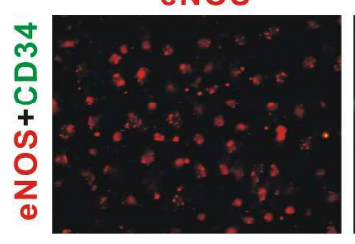

eNOS

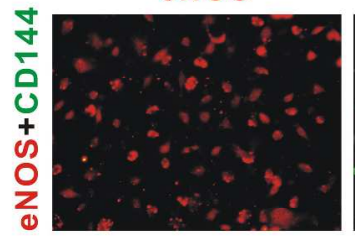

Flk-1

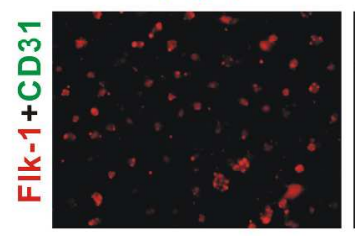

CD34

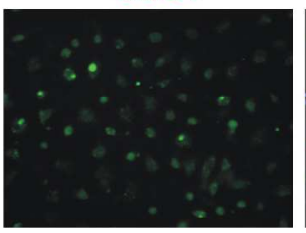

CD144

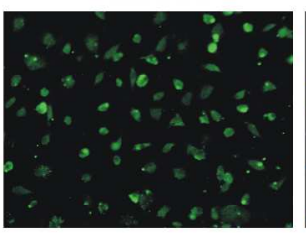

CD31

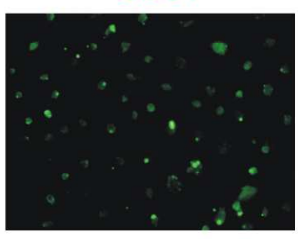

DAPI

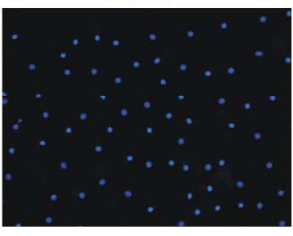

DAPI

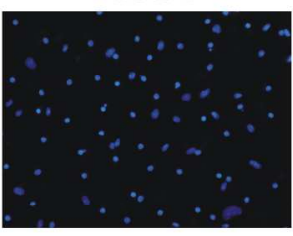

DAPI

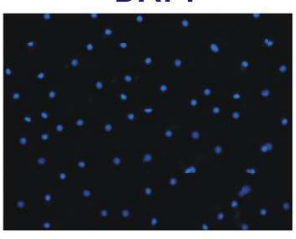

$\overline{100 u m}$

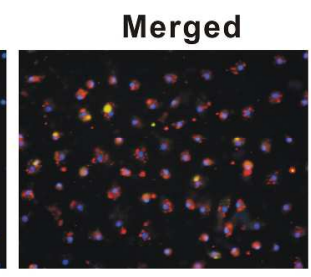

Merged

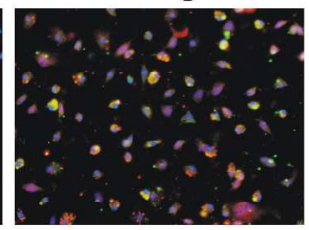

Merged

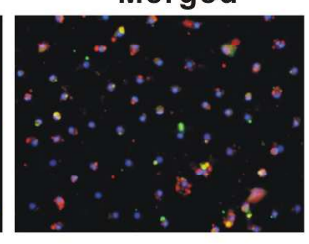

$100 \mathrm{um}$

Figure 1. Characterization of rat peripheral blood-derived endothelial progenitor cells (EPCs). A.Three-color fluorescent imaging indicating Dil-acetylated low-density lipoprotein (Dil-acLDL) incorporation (red) and FITC lectin (FITC-UEA-1) binding (green) by endothelial progenitor cells. Nuclei appear dark blue (DAPI). B. Immunofluorescence staining revealed the expression of CD34 (the stem cell marker), Flk-1, CD31, CD144 and eNOS (the endothelial cell markers) in EPCs. Nuclei were counterstained with DPI (blue).

Figure 1

$511 \times 622 \mathrm{~mm}(300 \times 300 \mathrm{DPI})$ 

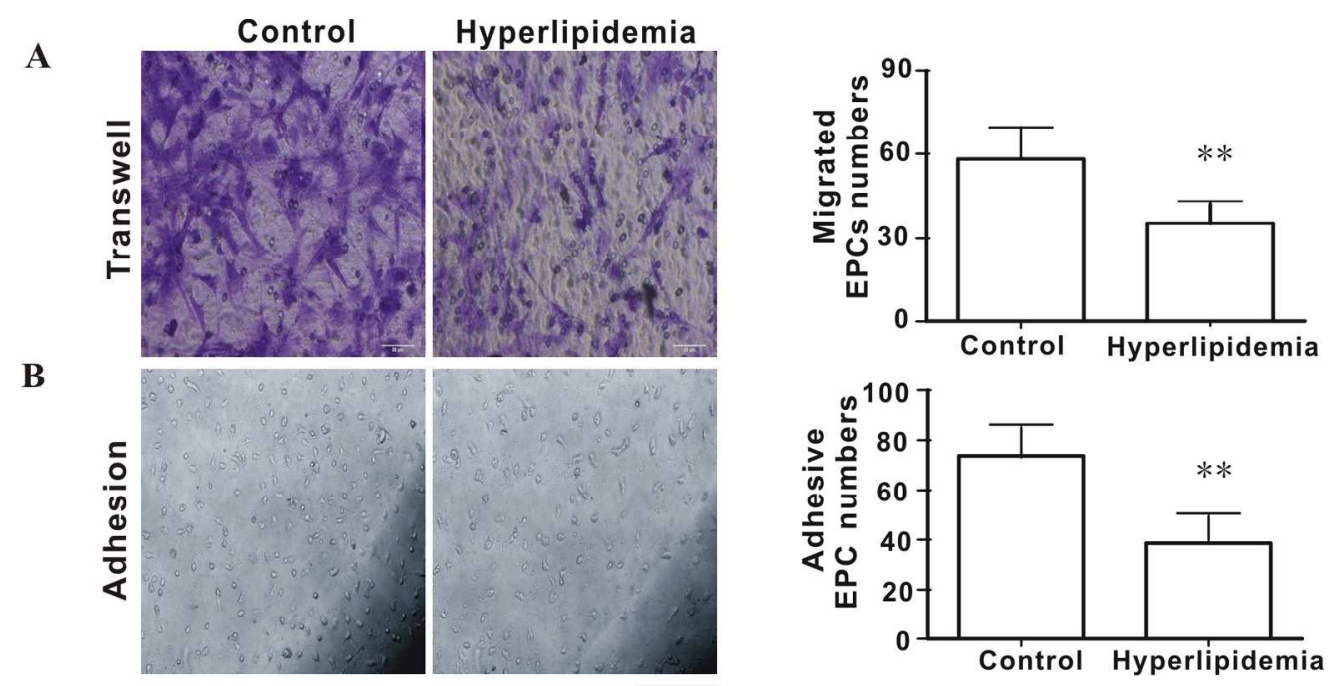

$100 \mathrm{um}$

Figure 2. Impaired functions of the circulating endothelial progenitor cells in hyperlipidemic rats. A. Left panel, representative images for EPCS migration (transwell) assay; right panel, the migrated EPC numbers. B. Left panel, representative images for EPCs adhesion assay; right panel, the adhesive EPC numbers. All values are expressed as mean $\pm S D\left(n=10\right.$ per group). ${ }^{* * P} \angle 0.01$ vs Control.

Figure 2

$170 \times 93 \mathrm{~mm}(300 \times 300 \mathrm{DPI})$ 
A

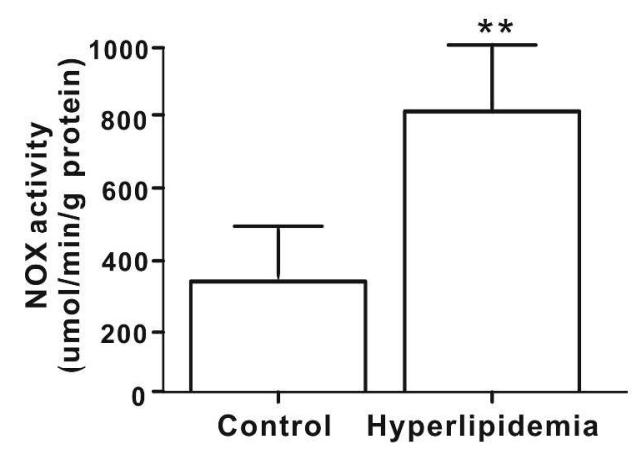

C

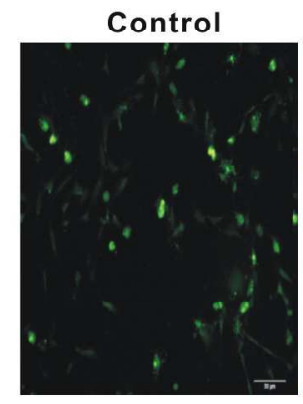

Hyperlipidemia

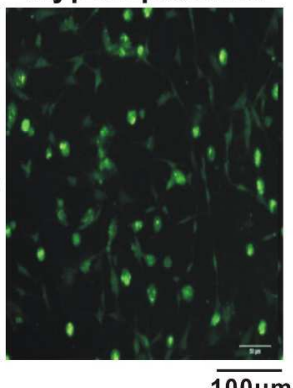

B
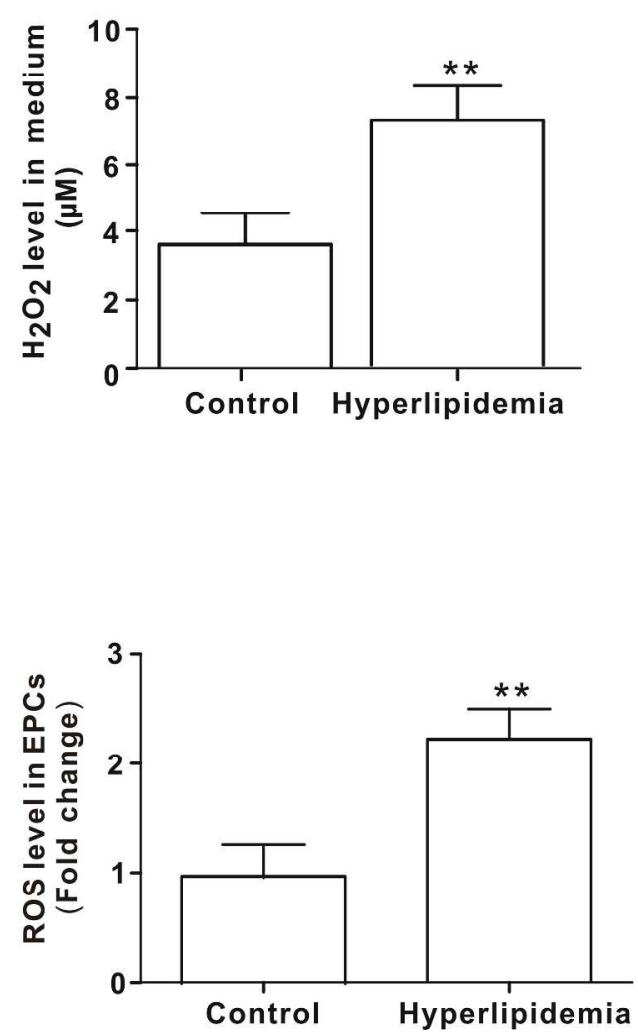

Figure 3. Elevation of NOX activity and ROS production in endothelial progenitor cells of hyperlipidemic rats. A. NOX activity in EPCs. B. H2O2 levels in culture medium. C. Left panel, Representative image of fluorescent signal of DCFH-DA for total ROS in EPCs; right panel, statistic value for fluorescent density. All values are expressed as mean \pm SD ( $n=10$ per group). NOX, NADPH oxidase; ROS, reactive oxidative species. ${ }^{*} \mathrm{P} \angle 0.01$ vs Control.

Figure 3

$281 \times 235 \mathrm{~mm}(300 \times 300 \mathrm{DPI})$ 
A

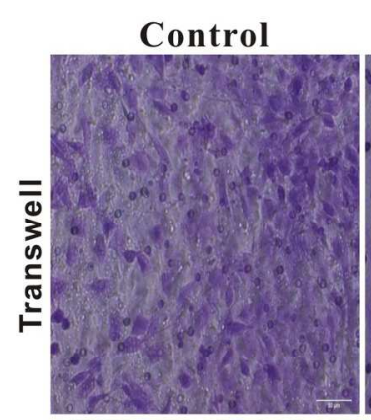

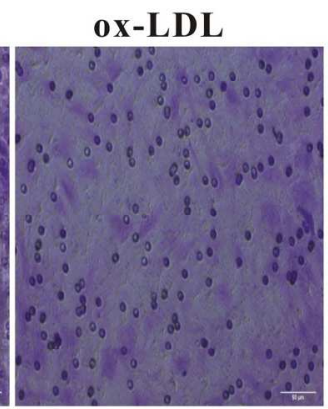

+VAS2870
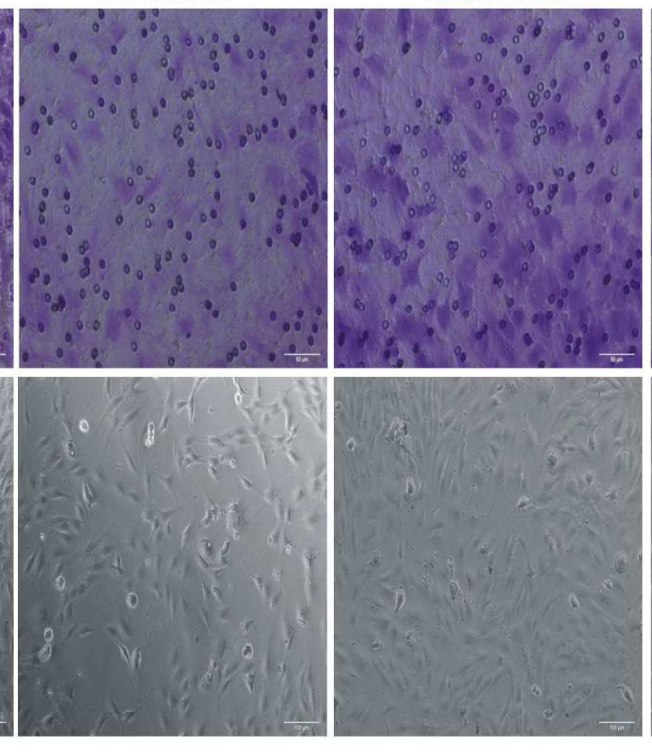

+Vehicle
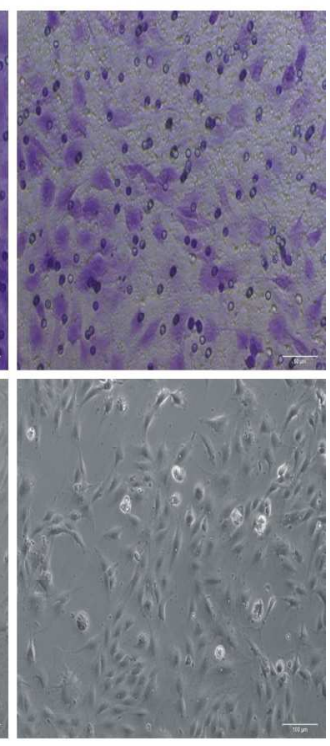

B

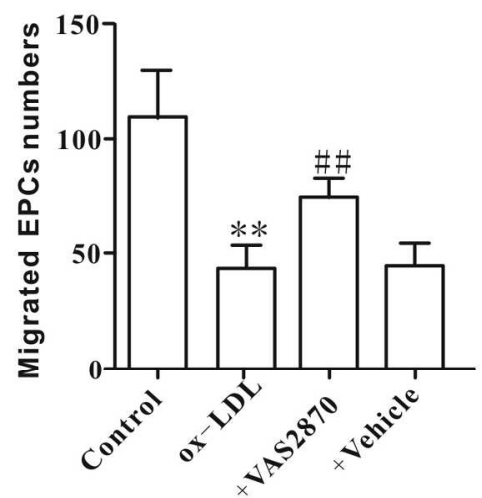

C

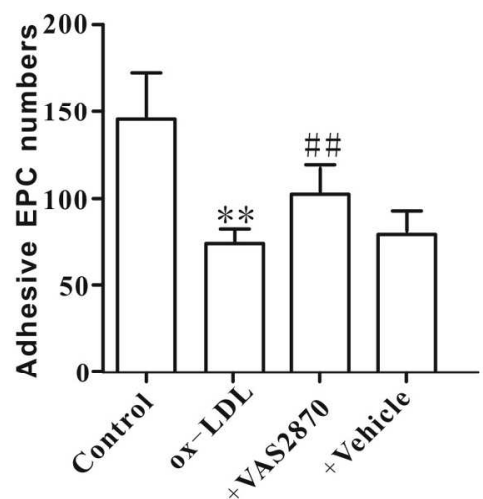

Figure 4. Impaired functions in ox-LDL-treated endothelial progenitor cells. A. Representative images for migration (transwell) and adhesion assays. B. The migrated EPC numbers $C$. The adhesive EPC numbers. All values are expressed as mean \pm SD ( $n=6$ per group). VAS2870: the NOX inhibitor; EPC, endothelial progenitor cell. ${ }^{* * P} \angle 0.01$ vs Control; \#\#P $\angle 0.01$ vs ox-LDL.

Figure 4

$178 \times 201 \mathrm{~mm}(300 \times 300$ DPI $)$ 
A

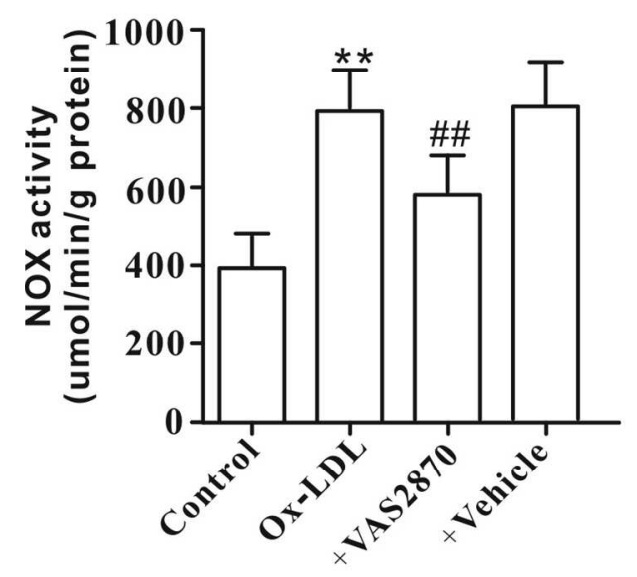

C

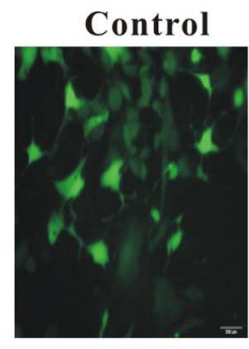

+VAS2870

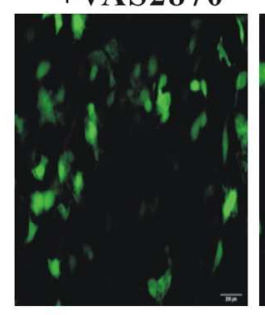

ox-LDL

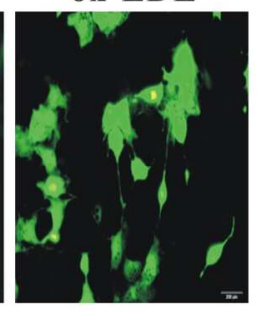

+Vehicle

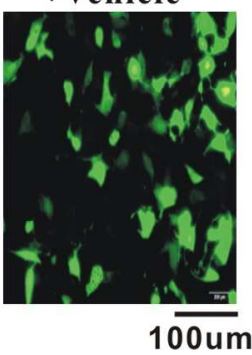

B
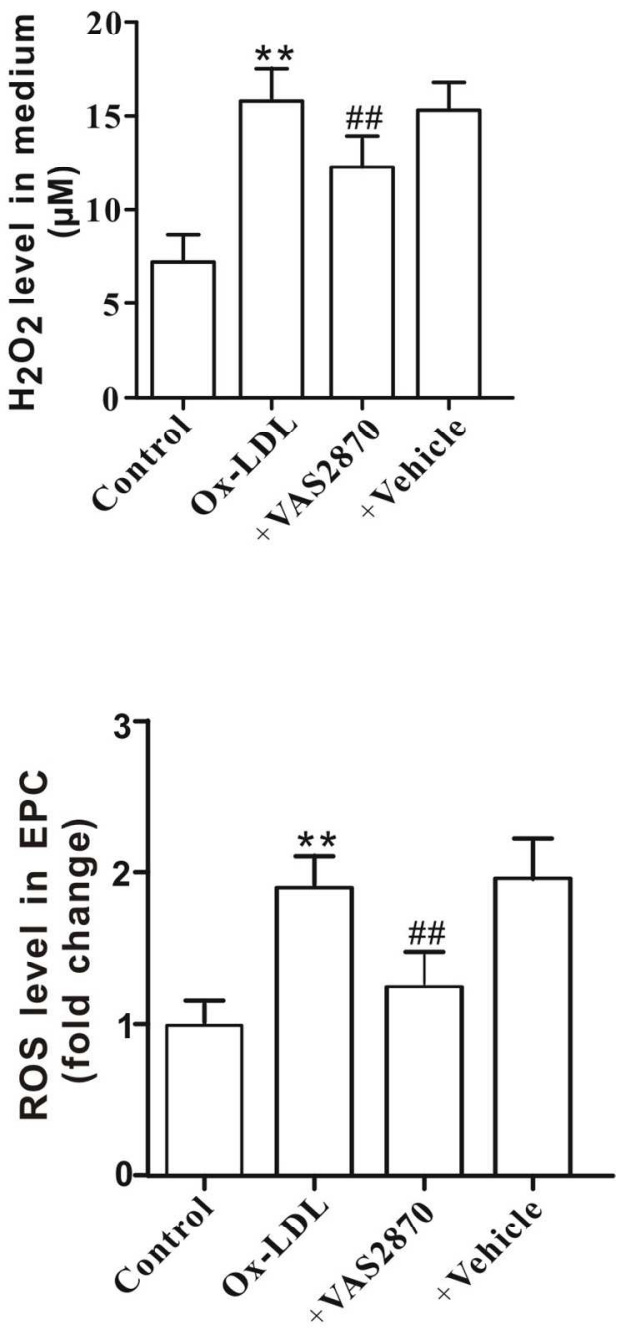

Figure 5. Elevation of NOX activity and ROS production in ox-LDL-treated endothelial progenitor cells. A. NOX activity in EPCs. B. H2O2 levels in EPCs. C. Left panel, Representative image of fluorescent signal of DCFH-DA for total ROS in EPCs; right panel, statistic value for fluorescent density. All values are expressed as mean \pm SD ( $n=6$ per group). NOX, NADPH oxidase; ROS, reactive oxidative species. $* * P \angle 0.01$ vs Control; \#\#P $\angle 0.01$ vs ox-LDL.

Figure 5

$145 \times 162 \mathrm{~mm}(300 \times 300 \mathrm{DPI})$ 